\title{
Improving tissue characterization, differentiation and diagnosis in gynecology with the narrow-band imaging technique: A systematic review
}

\author{
PANAGIOTIS PEITSIDIS ${ }^{1,2}$, NIKOLAOS VRACHNIS ${ }^{3,4}$, STAVROS SIFAKIS $^{5}$, CHRISTOS KATSETOS $^{1}$, \\ PANAGIOTIS TSIKOURAS ${ }^{6}$, NIKOLAOS ANTONAKOPOULOS ${ }^{3}$, \\ EVAGGELOS ALEXOPOULOS $^{7}$ and KONSTANTINOS KALMANTIS ${ }^{7}$
}

\footnotetext{
${ }^{1}$ National Public Health Organization of Greece, Department of Obstetrics and Gynecology, Tzaneio Hospital, 18536 Piraeus;

${ }^{2}$ Faculty of Midwifery, University of West Attica, 12243 Athens; ${ }^{3}$ rd Department of Obstetrics and Gynecology,

National and Kapodistrian University of Athens Medical School, Attikon Hospital, 12462 Athens, Greece;

${ }^{4}$ Department of Vascular Biology, Molecular and Clinical Sciences Research Institute, St. George's University of London,

London SW17 0QT, UK; ${ }^{5}$ Department of Obstetrics and Gynecology, Mitera Hospital, 71202 Heraklion;

${ }^{6}$ Department of Obstetrics and Gynecology, Democritus University of Thrace Medical School, 68100 Alexandroupolis;

${ }^{7}$ Department of Obstetrics and Gynecology, Alexandra Maternity Hospital, 11528 Athens, Greece
}

Received February 18, 2021; Accepted May 18, 2021

DOI: $10.3892 / \mathrm{etm} .2021 .10958$

\begin{abstract}
Narrow-band imaging (NBI), an on-demand, real-time endoscopic imaging technique, was developed to enhance visualization of the mucosal vascular network and surface texture. The present article provides a systematic review of studies that assessed the use of NBI in gynecological endoscopy. The following electronic databases were searched: PubMed (1950-2020), Google Scholar (2004-2020) and Cochrane Library (2010-2020). In the initial search, 3,836 entries were identified, of which 31 were finally included in the systematic review. Of the selected studies, $10(32 \%)$ were case reports, $19(61.2 \%)$ were prospective studies and $2(6.4 \%)$ were randomized controlled trials with control groups. The selected studies reported on the use of NBI in hysteroscopy, laparoscopy and colposcopy. It was revealed that NBI utilization in hysteroscopy increased the accuracy, sensitivity and specificity in detecting malignant and premalignant lesions. NBI improved the specificity and sensitivity in the detection of endometriotic lesions and cervical lesions. Conventional white light endoscopy in gynecology may be significantly improved by the use of NBI. Further studies with larger cohorts and improved design are required to achieve more reliable results. It is of special interest that utilization of
\end{abstract}

Correspondence to: Dr Panagiotis Peitsidis, National Public Health Organization of Greece, Department of Obstetrics and Gynecology, Tzaneio Hospital, Afentouli and Zanni Street, 18536 Piraeus, Greece

E-mail: peitsidiobgyn@gmail.com

Key words: hysteroscopy, laparoscopy, white light, narrow-band, endometriosis, colposcopy this method requires apparatus which is expensive; concerns are the long training and experience of staff required and the long learning curve.

\section{Introduction}

Narrow-band imaging (NBI), an on-demand, real-time, endoscopic imaging technique, was developed to enhance the visualization of the mucosal vascular network and surface texture for the purpose of improving tissue differentiation, characterization and diagnosis (1).

The interaction of particular tissue structures with light is wavelength-dependent and augmentation of particular mucosal features via NBI is achieved through observation of light transmission at selected wavelengths (or colors) (2).

In the NBI system, selective light transmittance is conducted via optical filtering of white light (WL). Specifically, NBI uses two discrete bands of light, a blue band at $415 \mathrm{~nm}$ and a green band at $540 \mathrm{~mm}$, to create a high-contrast image of the tissue surface, which allows enhanced visualization of blood vessels (3). The two bands correspond to the peak light absorption of hemoglobin, thus permitting NBI to visualize the blood vessels with greater clarity and accuracy on the surface of the analyzed tissues than observation with WL (3).

In clinical practice, there is currently widespread use of NBI to provide an improved examination of the gastrointestinal system, including the stomach and large intestine, esophagus and pharynx, as well as of the lungs, urinary tract and oropharynx. This technique, which has been termed 'optical biopsy', has brought achievements of earlier diagnosis by substantially improving the qualitative diagnosis of the depth and grade of invasion of atypical lesions (4).

The aim of the present study was to perform a systematic review of all available studies evaluating the use of NBI in 
gynecology clinical practice for the detection of benign and malignant lesions.

\section{Materials and methods}

Search strategy. The following electronic databases were searched: PubMed (1950-2021), Google Scholar (2004-2021) and Cochrane Library (2010-2021). The electronic literature search was mostly performed between January 2020 to February 2021. The search included the following medical subject headings or keywords: 'Narrow-band imaging' and 'gynecology'. The last search was performed on 08/02/2021.

The systematic review was performed and the flowchart diagram was drawn according to the Preferred Reporting Items for Systematic Reviews and Metanalyses statement (5).

Inclusion criteria. Full-text articles published in peer-reviewed journals and written in the English language were deemed eligible to be included in the review. Studies that did not fulfill the following criteria were excluded from the review: i) Conference abstracts and studies not providing sufficient clinical data; and ii) studies reporting narrow-band imaging utilization in animals, in surgical specimens or in an in vitro environment.

All types of studies were included, namely randomized controlled or observational studies, case series and case reports. The selected eligible articles were compared and discrepancies were resolved by discussion. The final decision on eligibility was made by the senior investigator (PP) whenever discrepancies had not been resolved through discussion.

Data extraction. Two authors (PP and KK) independently extracted information, while SS, CK, VA, PT and NA checked the extracted information and tabulated the data. NV checked the results and approved the study.

From the eligible studies, the following clinical data were obtained: Author and year of publication; the time period of enrolment of the study population; the country and city in which the study was performed; the type of study; the setting of the hospital (single- or multi-university study); the number of patients; the age of the patients; the inclusion criteria for surgery; the interventions performed; the endoscopic system used in each study; the outcome of the studies; and comments on different studies. The references of the selected studies were scrutinized for additional information not obtained by the initial search.

The Quality Assessment of Diagnostic Accuracy Studies 2 (QUADAS-2) tool was used to assess the quality of the primary diagnostic accuracy studies. Via this tool, risks for bias may be evaluated in the following four key domains: i) Selection of participants; ii) index (diagnostic) test; iii) reference standard; and iv) flow and timing of the study (6).

Through the use of the Case Report (CARE) guidelines checklist, the information quality of case reports was evaluated, specifically assessing the following items: Patient information, the presence of timeline information, diagnostic assessment, clinical findings, therapeutic intervention and outcomes $(7,8)$.

Statistical analysis. Data obtained from the selected studies were entered into an Excel v160 spreadsheet (Microsoft Corporation 2018). Descriptive statistical analyses was performed using SPSS version 23 (IBM Corporation) and
Excel version 16.0 (Microsoft Corporation, 2018). The number of publications were calculated per year and per country, and the median age of participants in the selected studies was also calculated.

\section{Results}

Literature search and selection. The literature search identified a total of 3,836 studies, i.e., 3,800 articles through Google Scholar, 32 articles through the PubMed database and four through the Cochrane Library database. The flowchart of the study selection process is displayed in Fig. 1. After removing duplicates and irrelevant articles, 256 articles were considered for further evaluation. Further assessment excluded 215 articles for various reasons (non-eligible, non-English literature, animal studies and articles providing insufficient information). Finally, 31 studies reporting on a total of 3,128 female patients were included in the review.

Of the selected studies, 10 (32\%) were case reports (9-18), 19 $(61.2 \%)$ were prospective studies $(11,12,18-32)$ and two studies $(6.4 \%)$ were randomized controlled trials $(33,34)$. The selected studies had publication dates ranging from 2007 to 2020 .

The frequency of publications per year and the percentage of publications by country are presented in Fig. 2. The years with the highest frequency of publications were 2010 and 2011 with 5 studies produced each year and the country which produced the highest number of studies was Japan with 11 studies $(13,18,21,22,28,31-33,35)$, followed by Italy with 8 studies $(9,10,15-17,19,20,36)$.

Study properties. A total of 11 studies described the use of NBI in hysteroscopy for the detection of endometrial pathologies $(9,10,19-25,36,37)$ and another 11 studies reported the use of NBI in laparoscopy for the detection of peritoneal endometriosis (11-13,26-30,33,34,37,38). Furthermore, three articles dealt with the use of NBI in digital colposcopy for the detection of lower genital tract pathologies $(14,31,32)$ and three articles reported on the use of NBI in laparoscopy for the detection of peritoneal metastases associated with ovarian cancer and other gynecological malignancies (15-17).

NBI in endometrial pathology. Details on the selected studies reporting the use of NBI in hysteroscopy are presented in Table I. A total of 11 studies were included in the review, dating from 2009-2020 with a combined cohort of 2,424 female patients $(9,10,19-25,36,37)$. Of these, eight studies were prospective studies (Canadian Task Force classification, II-2) $(19,23,36)$ and two studies were case reports $(9,10)$. All studies were designed in university settings, two studies were performed in multicentric university settings $(20,36)$ and two studies were reported at international congresses as conference reports $(10,24)$. All of the patients presented with abnormal uterine bleeding (AUB). The median age of the patients was $45.5 \pm 10.5$ years. A total of four studies were performed in outpatient office settings using a vaginoscopic approach without any general anesthesia $(19,21,23,36)$. Conventional hysteroscopy under general anesthesia was performed in seven studies $(10,24,25)$. Operative hysteroscopy was performed in all cases and histology specimens were obtained in each case. Olympus Exera II (Olympus Corporation) was the main video system used in the majority of studies. 


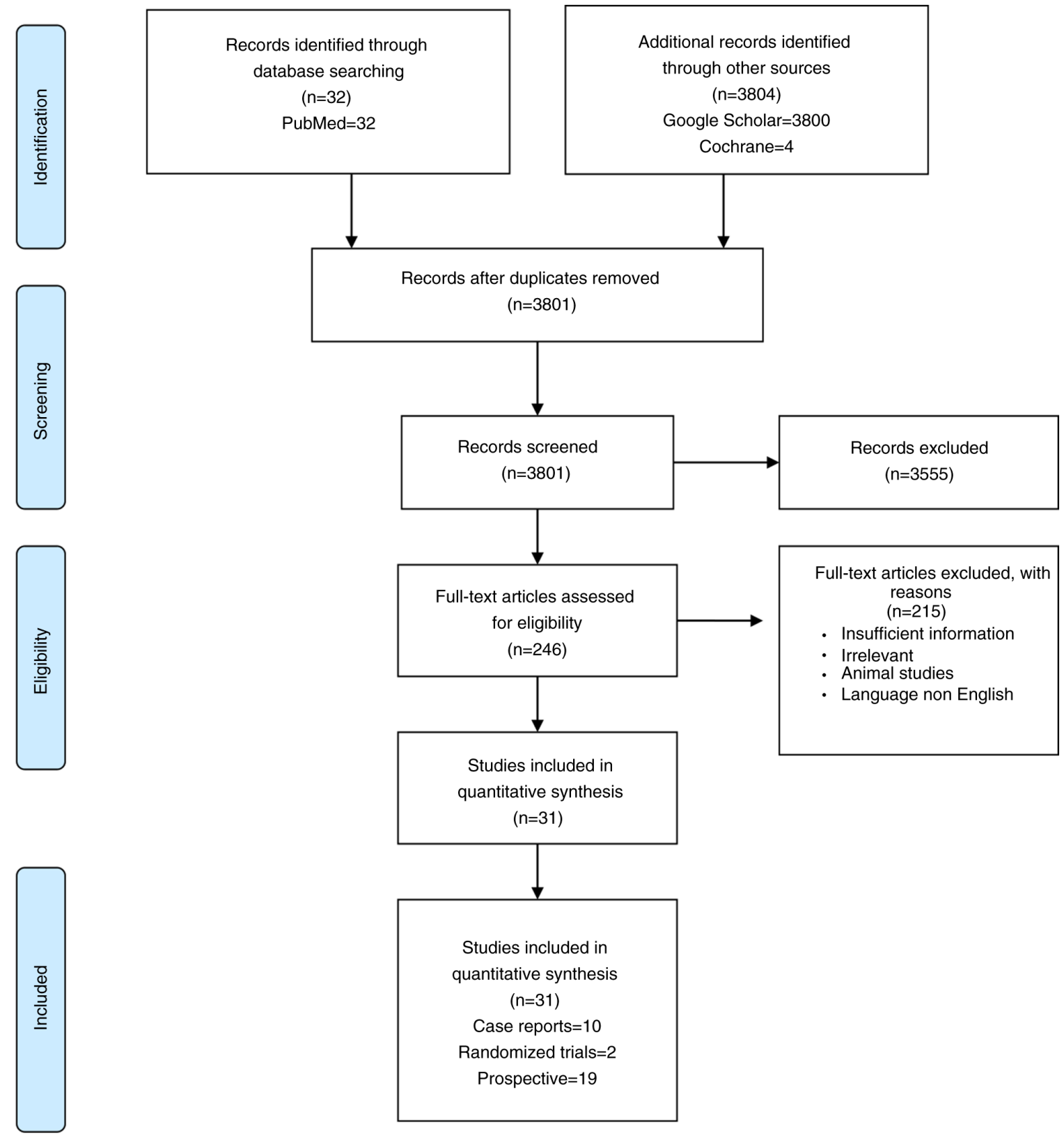

Figure 1. Flowchart diagram of the study selection for systematic review according to the Preferred Reporting Items for Systematic Reviews and Metanalyses guidelines.

NBI hysteroscopy demonstrated increased sensitivity compared with WL imaging hysteroscopy for the detection of endometrial cancer in four studies $(20,23,24)$.

The sensitivity of NBI and WL hysteroscopy for endometrial cancer reported by individual studies was 78.6 vs. $63.7 \%, \mathrm{P}<0.001$ (23), 93 vs. $81 \%, \mathrm{P}<0.05$ (36), 94.7 vs. $84.4 \%$, $\mathrm{P}<0.05$ (20) and 98.1 vs. $73.6 \% \mathrm{P}<0.001$ (24). Increased sensitivity of NBI vs. WL for the detection of atypical endometrial hyperplasia - high-risk hyperplasia $(\mathrm{HRH})$ was reported in five studies $(10,20-23)$. The reported sensitivity of NBI vs. WL for HRH was 60 vs. $20 \%, \mathrm{P}<0.005$ (36), 78.38 vs. $64.86 \%$, $\mathrm{P}<0.005$ (20), 78.6 vs. $63.7 \%, \mathrm{P}<0.001$ (23), 93.5 vs. $82.6 \%$, $\mathrm{P}=0.006$ (24) and 97.2 vs. $82.6 \%, \mathrm{P}<0.005$ (21).

None of the 11 studies reported any adverse effects or complications related to the surgical techniques in all instances.
NBI in endometriosis. Details on the selected studies reporting on the use of NBI in laparoscopy for the detection of peritoneal endometriosis are provided in Table II. A total of 11 studies were included in the present review, dating from 2007 to 2019 with a population of 626 female patients in total $(11-13,26-30,33,34,38)$. The mean age was $35.5 \pm 3.6$ years. A total of three studies were case reports $(11,12,29)$, three were studies with a randomized design $(13,34,38)$ and five were had a prospective design lacking randomization (26-29,33). In all studies, laparoscopy with a 0 -degree 10-mm scope was performed, except for two studies where intestinal endoscopy was utilized (13), while one study reported on the use of a 0 -degree 12-mm scope (30). NBI improved the detection rate by $53 \%$ for smaller endometriotic lesions previously not detected on WL imaging laparoscopy (26). Another study reported that $82.7 \%$ of endometriotic lesions were detected using NBI compared with $55.9 \%$ of lesions detected 

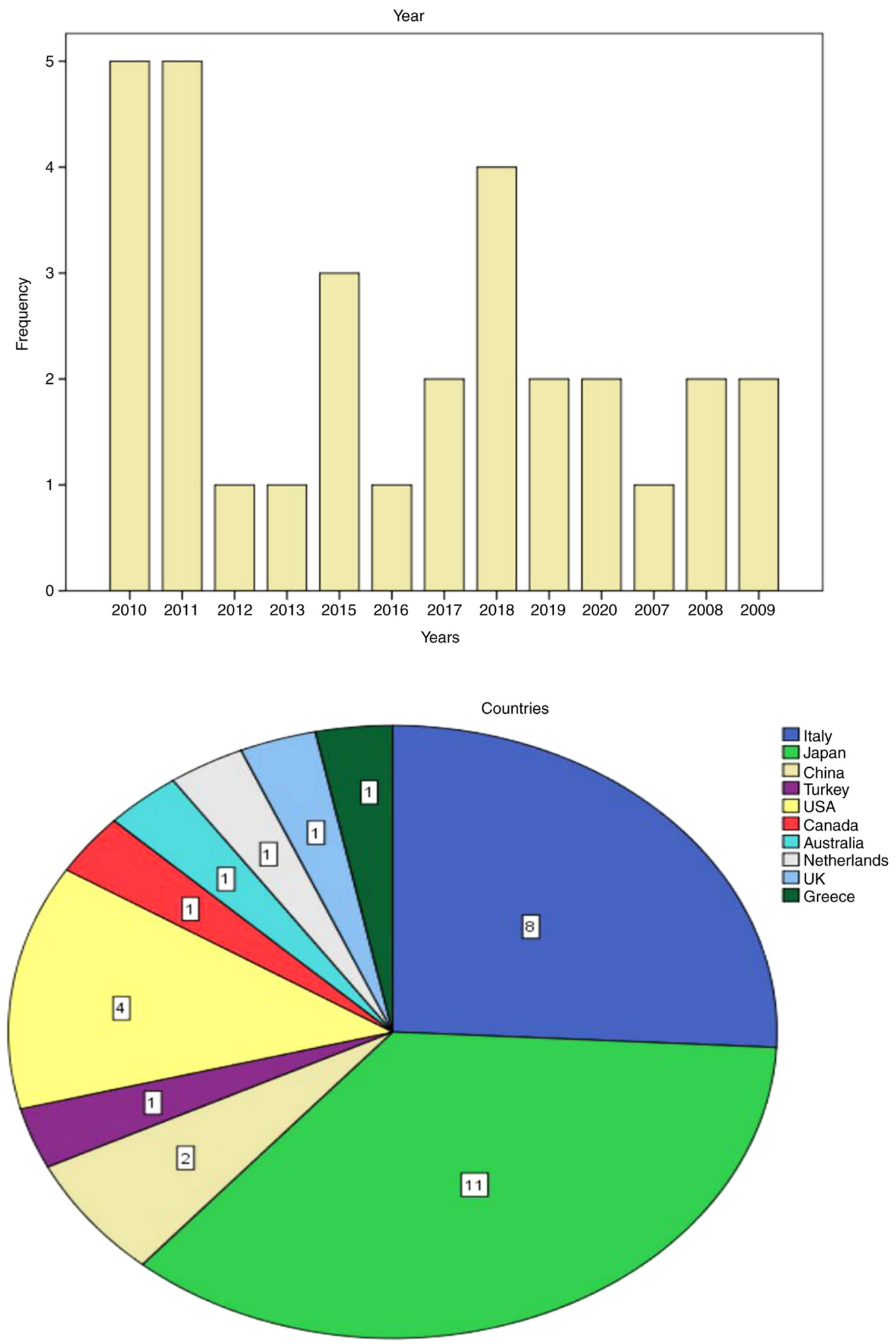

Figure 2. Frequency of publications per year and per country. A Histogram and pie chart are provided in order to present the frequency of selected publications per country and per year.

by WL imaging (27). The detection rate of endometriotic lesions with NBI was $100 \%$ in a randomized controlled trial with 167 female patients (34). Another randomized controlled trial assessed quality of life in two study groups: One group underwent laparoscopy with WL and the other with NBI. No difference in pain and quality of life was observed between the two study groups (34). When combined with WL, NBI was reported to provide an additional predictive value of $86 \%$ for the detection of endometriotic lesions, if a positive diagnosis was made under WL imaging alone (29). NBI and WL combined with $3 \mathrm{D}$ imaging was able to increase the sensitivity rate up to $91 \%$ (13).

Olympus Exetera (Olympus Corporation) was the system used for NBI in all of the studies. None of the 11 studies reported any adverse effects or complications related to the surgical techniques. 


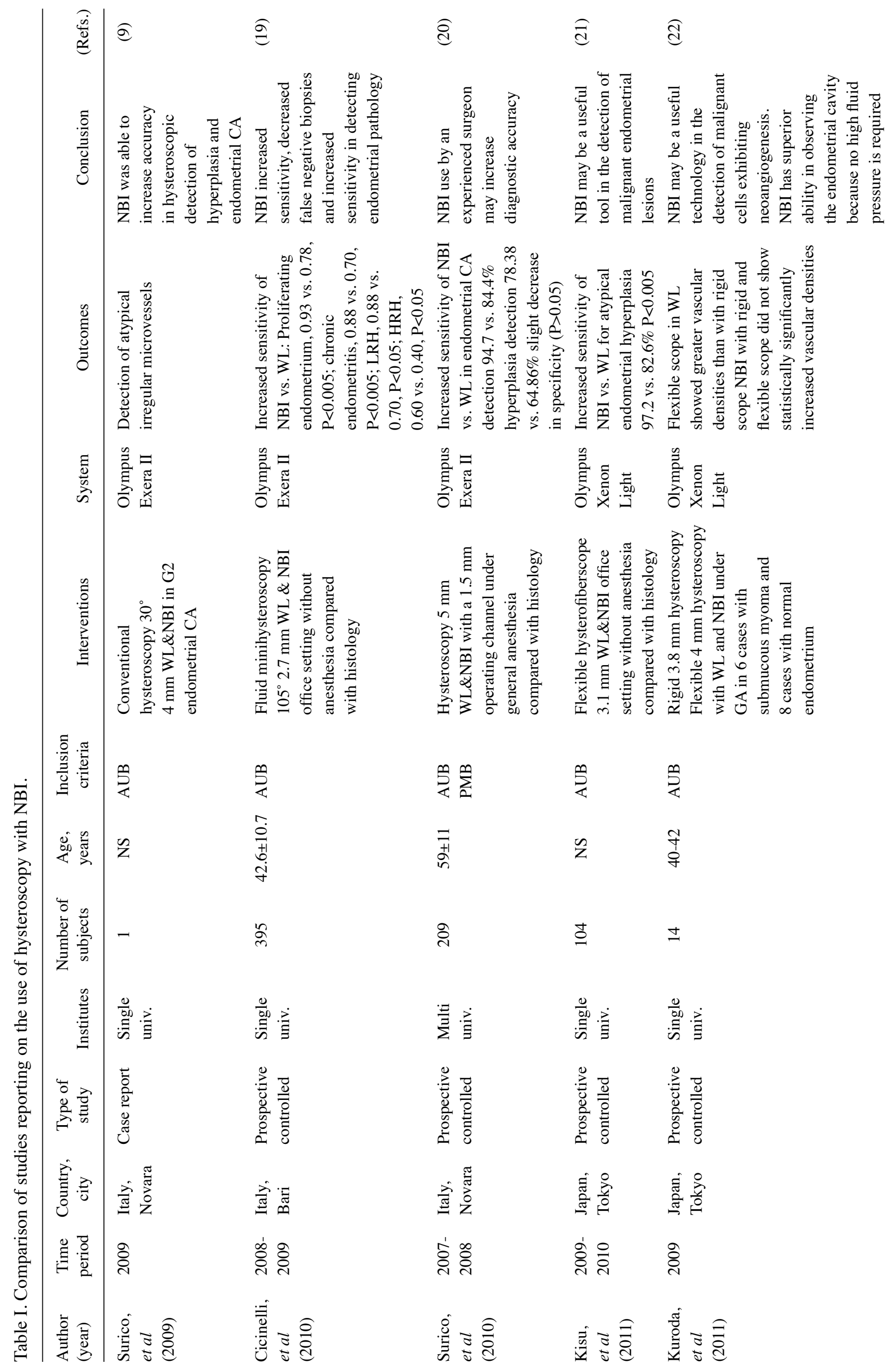




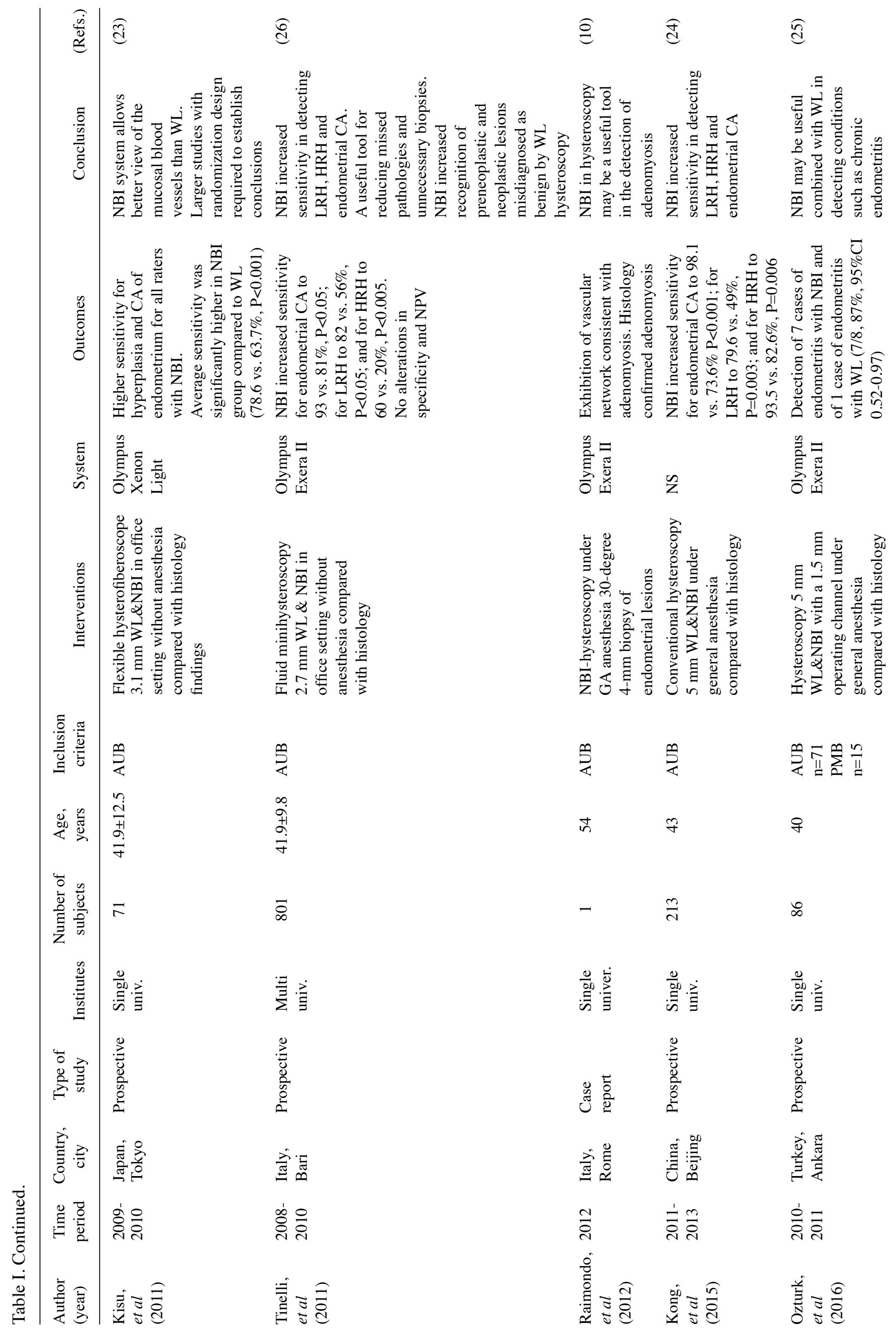


NBI in cervical pathology and peritoneal implants. Details on the selected studies reporting on the use of NBI in laparoscopy for the detection of cervical lesions and gynecological malignancies are listed in Table III. A total of 9 studies were included in the review, dating from 2010 to 2020 . Of these, four studies were prospective studies $(31,32,35,39)$ and five studies were case reports (14-18). The total number of patients was 156 and the mean age was $43.3 \pm 17.25$ years. Uterine cervical pathology was investigated with NBI in five studies $(14,18,31,32,35)$ and peritoneal implants were investigated with NBI in four studies $(15-17,39)$.

The vascular pattern of lesions was examined in 21 patients with early and in situ cervical adenocarcinoma using NBI (13). The authors classified the colposcopic lesions according to the pattern described by Wright (40). The vascular pattern was visualized in 18 patients $(86 \%)$; the authors concluded that NBI colposcopy depicts the vascular pattern on the cervix in early glandular disease better than conventional colposcopy. Nishiyama et al (32) proposed a novel microvascular classification. They used flexible magnifying endoscopy with NBI in 10 patients with cervical lesions; the detection rate was $90 \%(9 / 10)(32)$. NBI assisted in the detection of a rare melanoma of the cervix in a case report (14). Furthermore, NBI was utilized in laparoscopy for the detection of peritoneal metastasis in a series of 95 female patients undergoing surgery for various gynecological malignancies, as reported by Aloisi et al (39). They determined that NBI increased the number of the detected peritoneal abnormalities; however, no statistically significant differences were observed in the identification of histologically confirmed metastatic disease $(\mathrm{P}=0.18)(39)$.

A significantly greater number of peritoneal abnormalities were identified with NBI than with standard WL imaging. However, no statistically significant differences were observed in the identification of histologically confirmed metastatic disease (39). In addition, NBI proved to be useful in the laparoscopic detection of early peritoneal implants in three case reports (15-17). Kobara et al (18) indicated that NBI increased the sensitivity, specificity, accuracy and positive predictive value for the detection of cervical intraepithelial lesions-3 (CIN-3) in comparison with conventional colposcopy. NBI with a gastroscope assisted the detection of high-grade cervical intraepithelial lesions in two patients, while these lesions were not identified by conventional colposcopy $(18,35)$. None of the nine studies reported any adverse effects or complications related to the surgical techniques.

Quality assessment of case reports. The CARE guidelines were followed to perform the quality assessment of the case reports (7). A total of 10 case reports were assessed for their quality (9-18). The results are presented in Fig. 3. Approximately $30 \%$ of the case reports had a low quality in terms of presentation of demographic information. In total, $55 \%$ of the case reports included had low quality regarding the presentation of the patients' history with timeline information. Furthermore, $45 \%$ of the case reports were of poor quality in terms of information about differential diagnosis.

Quality assessment with QUADAS-2. The QUADAS-2 tool was used to assess the risk of bias in four domains. The results are presented in Fig. 4. In total, 19 studies were assessed. Of 


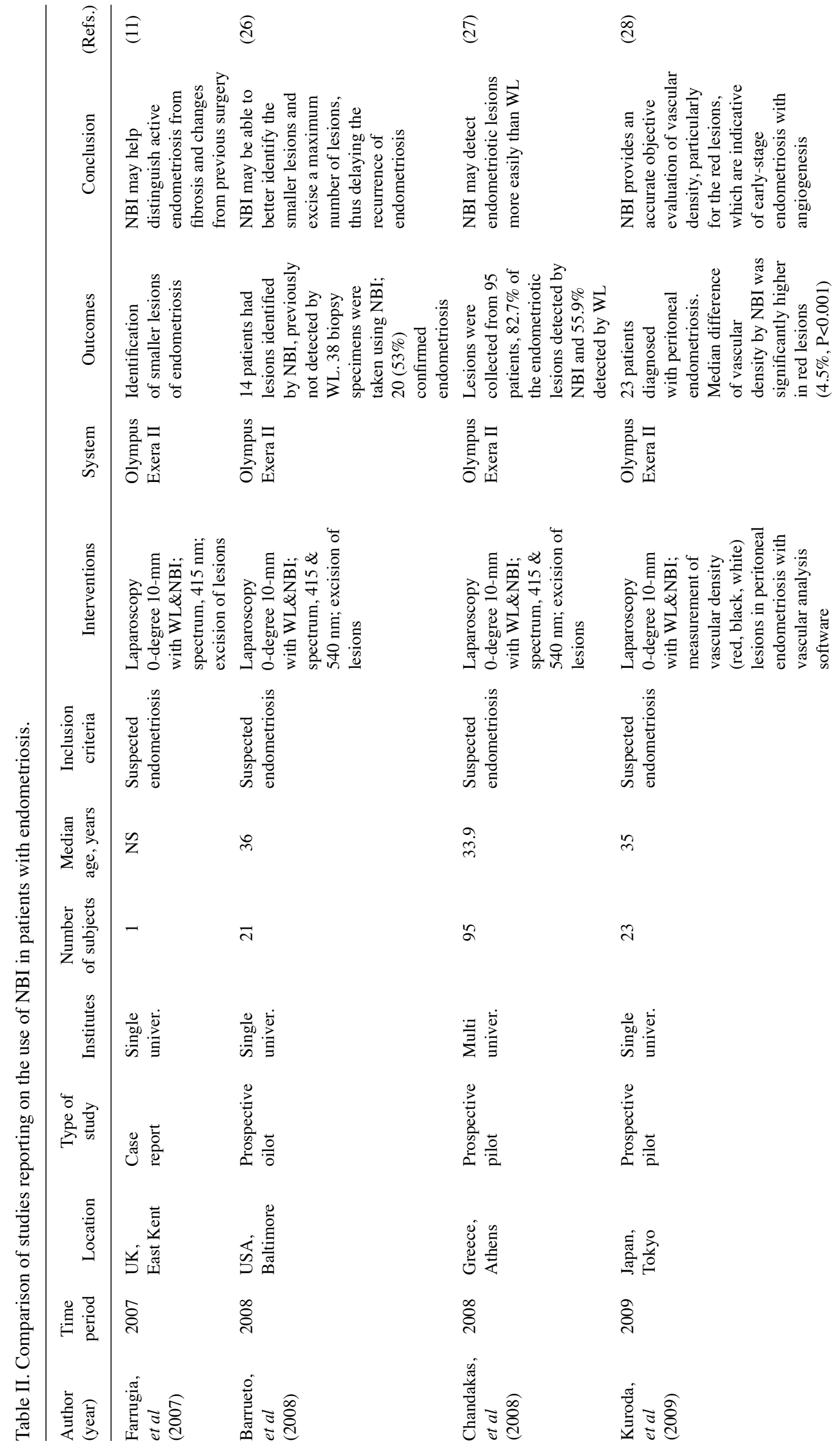









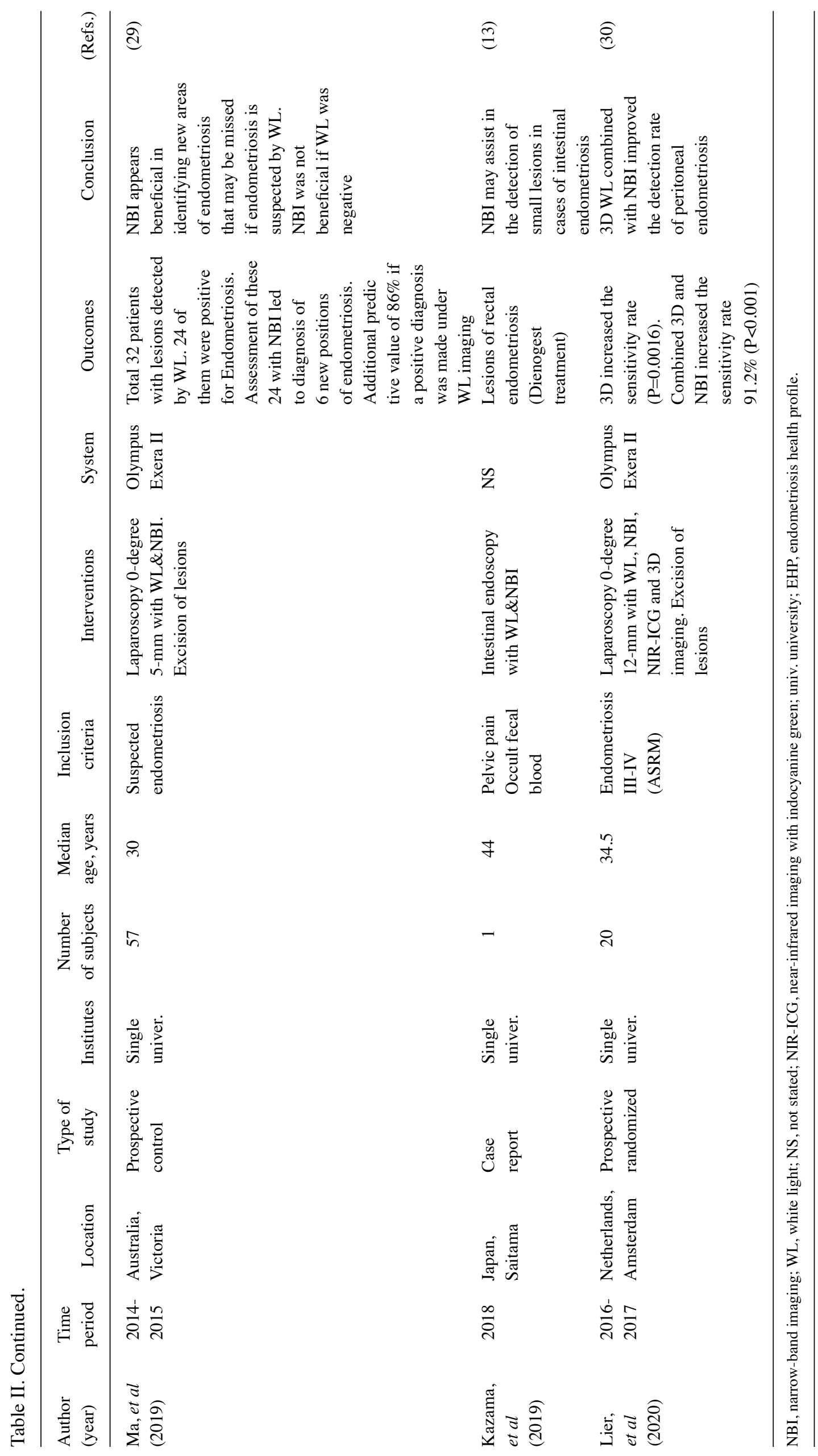




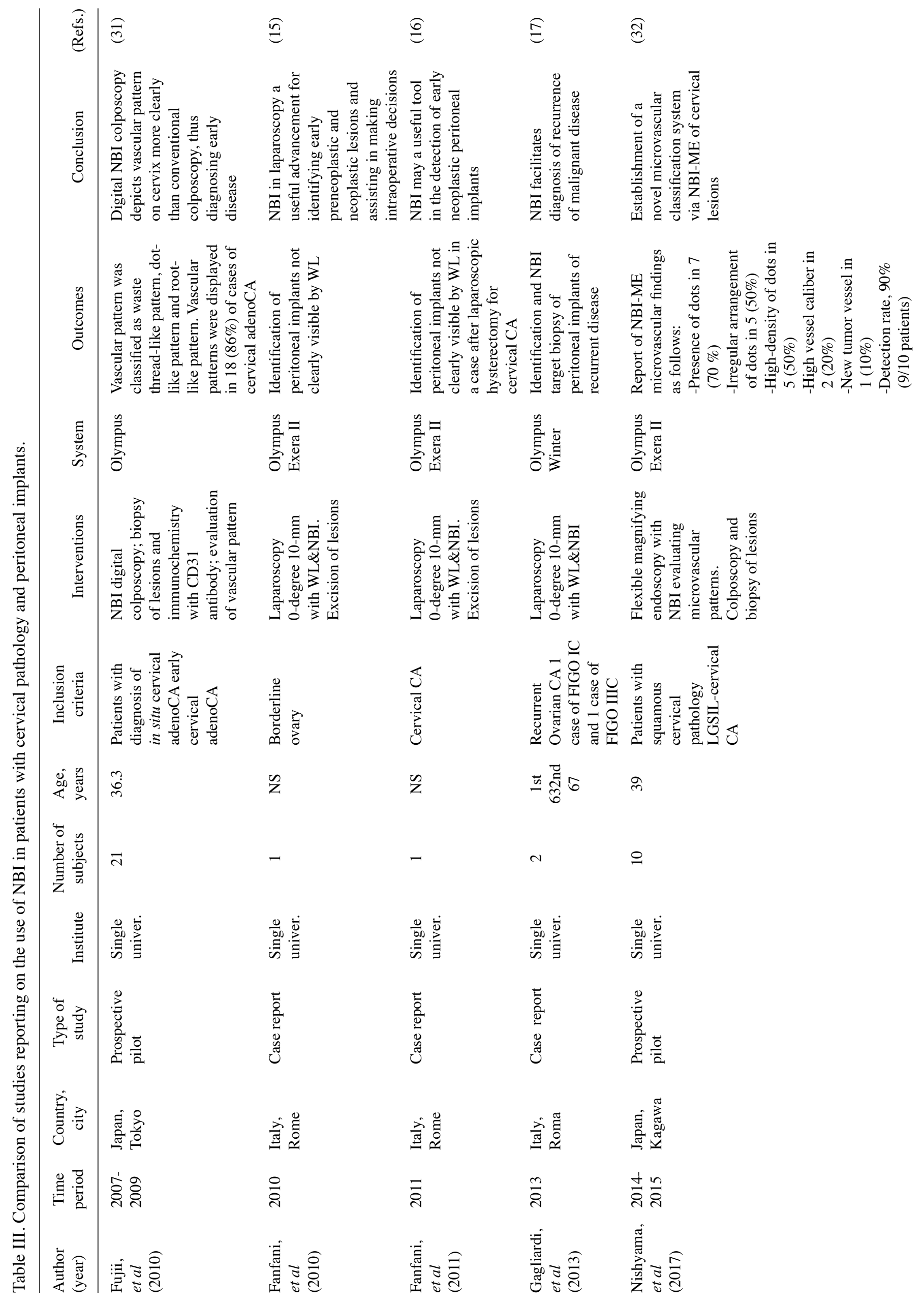




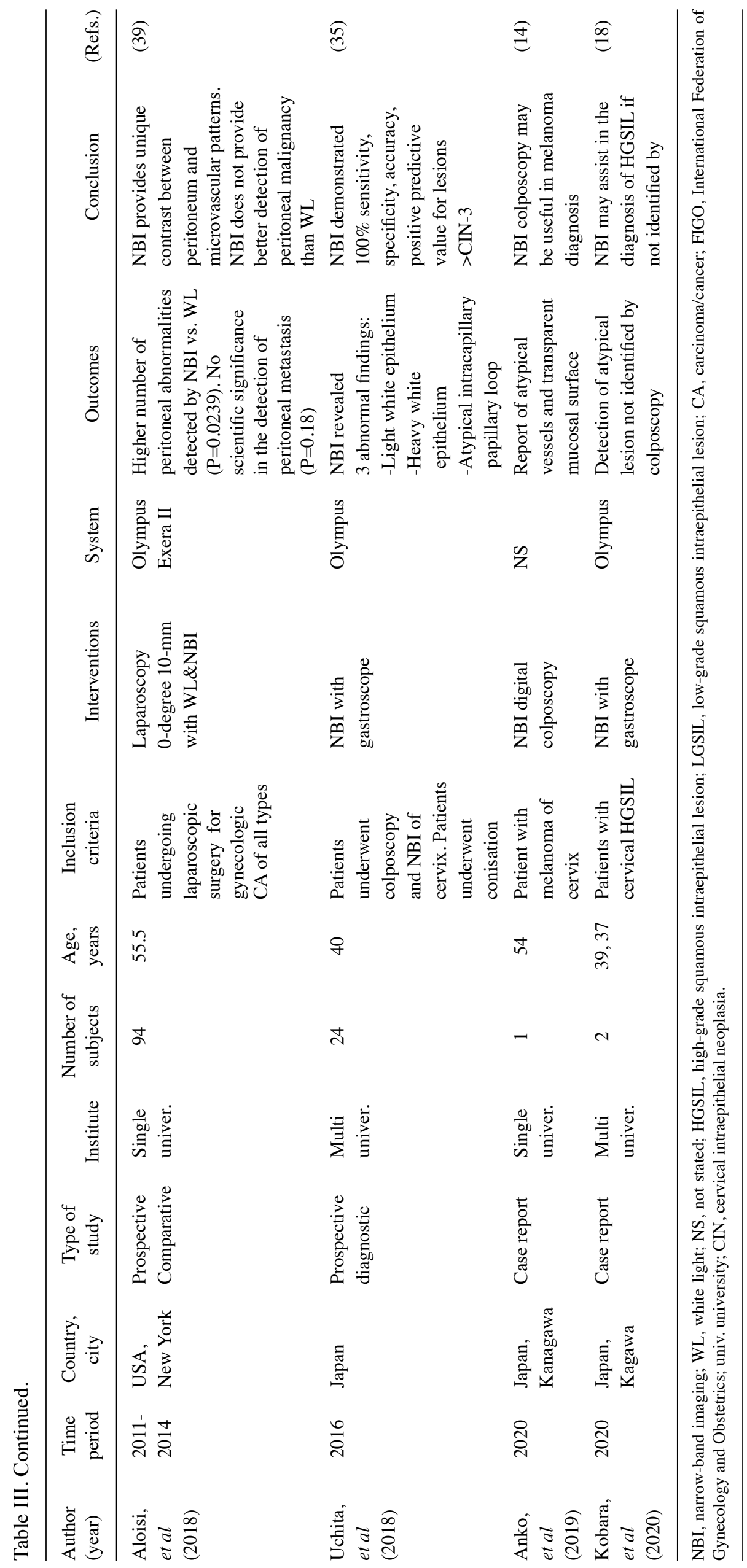




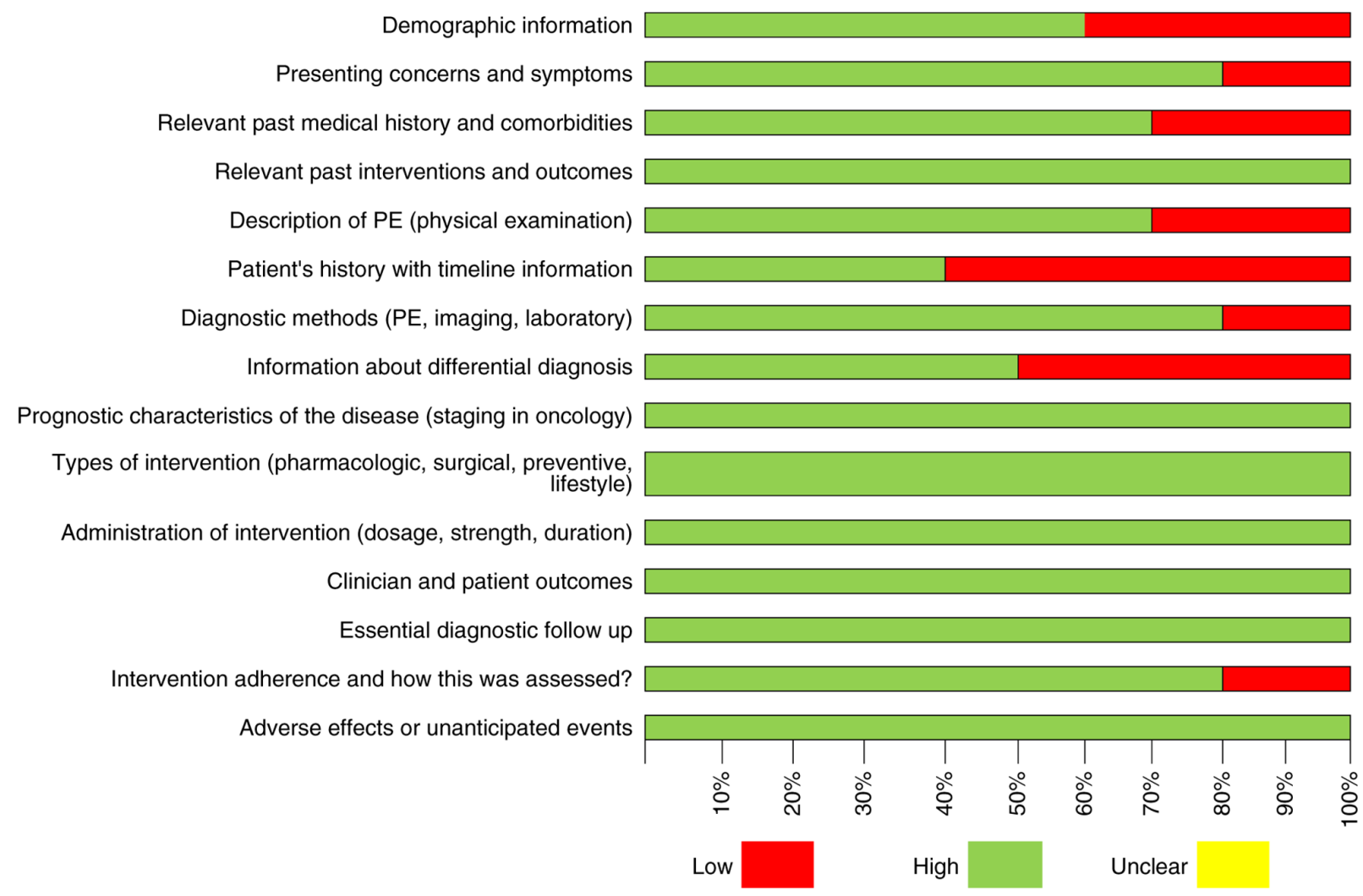

Figure 3. Quality assessment of case reports according to Case Report guidelines. Quality is rated as high, low or unclear.

these, 17 had a prospective design lacking randomization and two were randomized controlled trials with control groups. A total of 9 studies were indicated to have a high risk of bias $(24,25,27,29,30-32,34,36)$ and 10 studies were determined to have a low risk of bias (19-21,23,28,30,32,37-39).

NBI in gynecological endoscopy and special precautions in view of the Coronavirus Disease 2019 (COVID-19) pandemic. The outbreak of the COVID-19 pandemic caused by the Severe Acute Respiratory Syndrome Coronavirus-2 (SARS-CoV-2) has produced a public health emergency of international concern, with all of the data demonstrating that the spread of the virus mainly occurs via respiratory droplets during close contact $(41,42)$. During this ongoing pandemic, recommendations regarding laparoscopy and endoscopy with NBI should follow the general international recommendations for laparoscopy.

However, the potential risk of infection through endoscopically generated bioaerosols may possibly be increased as a result of three important factors pertaining to laparoscopy. These are the following: i) Use of gas insufflation during entry and intra-operatively; ii) generation of bioaerosols via electrosurgery, which is a cornerstone of endoscopy; and iii) the potential for gas leaks in the operation room, which may lead to elevated viral loads in the air $(41,42)$. Precautions should be taken during gynecological endoscopy with NBI, adhering to the international recommendations concerning SARS-CoV-2 (41-43).

Regarding elective surgery, it is recommended that universal testing for SARS-CoV-2 infection is conducted whenever possible within $40 \mathrm{~h}$ preceding surgery. Should a case of SARS-CoV-2 be positively confirmed, it is advisable to delay surgery, although exceptions may be made for urgent cases, e.g. malignancies.

It has been reported that laparoscopies have a larger potential for aerosol spread than hysteroscopies (41-43), while laparotomies have a lower risk of aerosol spread in comparison to laparoscopies (41-43). However, endoscopy is generally preferred due to the one-day stay and the smaller exposure to a hospital environment.

Strict safety measures regarding the pressure of the gas and pressure of the fluid should be applied to minimize the possibility of spread. In cases where local anesthesia may be used, it is expected to be beneficial for the patient, minimizing the risk of infection, which may occur after the intubation and the extubation procedure.

Further guidelines issued by the different medical associations such as International Society for Gynecologic Endoscopy (ISGE; www.isge.org) and American Society of Gynecologic Laparoscopists (AAGL; https://www.aagl.org/) $(42,43)$ will provide additional pertinent and vital data during the current fight against the pandemic.

Strengths and limitations. NBI, which is now regularly used in gynecology, is a relatively new optical technology and the present systematic review is, to the best of our knowledge, the most updated and extensive of its kind that has been provided on this method to date. Due to the relatively small number of randomized controlled trials, observational studies were included in spite of providing a lower level of evidence. While precise and clear inclusion criteria were employed for the preparation of the present review, the small sample size of the trials included and the overall absence of definitions of primary 


\begin{tabular}{|c|c|c|c|c|c|c|c|c|}
\hline & & & Risk of & Bias & & Applicability & Concerns & \\
\hline & & Patient & Index & Reference & Flow and & Patient & Index & Reference \\
\hline Study authors & Year & Selection & Test & Standard & Timing & Selection & Test & Standard \\
\hline Barrueto ${ }^{26}$ & 2008 & $\mathbf{L}$ & $\mathbf{L}$ & $\mathbf{L}$ & $\mathrm{H}$ & $\mathbf{L}$ & $\mathbf{L}$ & $\mathbf{L}$ \\
\hline Chandakas $^{27}$ & 2008 & U & $\mathbf{U}$ & $\mathrm{H}$ & $\mathrm{H}$ & $\mathbf{U}$ & $\mathbf{U}$ & H \\
\hline Kuroda $^{28}$ & 2009 & $\mathbf{L}$ & $\mathbf{L}$ & $\mathbf{L}$ & $\mathbf{L}$ & $\mathbf{L}$ & $\mathbf{L}$ & $\mathbf{L}$ \\
\hline Fujiji & 2010 & $\mathbf{L}$ & $\mathbf{L}$ & $\mathrm{H}$ & $\mathbf{L}$ & $\mathbf{L}$ & $\mathbf{L}$ & $\mathbf{L}$ \\
\hline Cicinelli ${ }^{19}$ & 2010 & $\mathbf{L}$ & $\mathbf{L}$ & $\mathbf{L}$ & $\mathbf{L}$ & $\mathbf{L}$ & $\mathbf{L}$ & $\mathbf{L}$ \\
\hline Surico $^{9}$ & 2010 & $\mathbf{L}$ & $\mathbf{L}$ & $\mathbf{L}$ & $\mathbf{L}$ & $\mathbf{L}$ & $\mathbf{L}$ & $\mathbf{L}$ \\
\hline $\mathrm{Kisu}^{21}$ & 2011 & $\mathbf{L}$ & $\mathbf{L}$ & $\mathbf{L}$ & $\mathbf{L}$ & $\mathbf{L}$ & $\mathbf{L}$ & $\mathbf{L}$ \\
\hline Kuroda ${ }^{22}$ & 2011 & $\mathbf{L}$ & $\mathbf{L}$ & $\mathbf{L}$ & $\mathbf{L}$ & $\mathbf{L}$ & $\mathbf{L}$ & $\mathbf{L}$ \\
\hline $\mathrm{Kisu}^{23}$ & 2011 & $\mathbf{L}$ & $\mathbf{L}$ & $\mathbf{L}$ & $\mathbf{L}$ & $\mathbf{L}$ & $\mathbf{L}$ & $\mathbf{L}$ \\
\hline Tinelli $^{36}$ & 2011 & U & $\mathbf{L}$ & $\mathbf{L}$ & $\mathrm{H}$ & $\mathbf{U}$ & $\mathbf{L}$ & $\mathbf{L}$ \\
\hline Kong $^{24}$ & 2015 & $\mathbf{L}$ & $\mathbf{L}$ & $\mathbf{L}$ & $\mathrm{H}$ & $\mathbf{L}$ & $\mathbf{L}$ & H \\
\hline Gallichio ${ }^{38}$ & 2015 & $\underline{L}$ & $\mathbf{L}$ & $\mathbf{L}$ & $\mathbf{L}$ & $\mathbf{L}$ & $\mathbf{L}$ & $\mathbf{L}$ \\
\hline Ozturk ${ }^{24}$ & 2016 & $\mathbf{L}$ & $\mathbf{L}$ & $\mathbf{L}$ & $\mathrm{H}$ & $\mathrm{H}$ & $\mathbf{L}$ & $\mathbf{L}$ \\
\hline Barrueto ${ }^{34}$ & 2015 & U & $\mathbf{L}$ & H & $\mathbf{L}$ & $\mathbf{L}$ & $\mathbf{L}$ & $\mathbf{L}$ \\
\hline Nishyama $^{32}$ & 2017 & $\mathbf{L}$ & $\mathbf{L}$ & $\mathbf{L}$ & $\mathbf{L}$ & $\mathbf{L}$ & $\mathbf{L}$ & $\mathbf{L}$ \\
\hline Aloisis ${ }^{39}$ & 2018 & $\mathbf{L}$ & $\mathbf{L}$ & $\mathbf{L}$ & $\mathbf{L}$ & $\mathbf{L}$ & $\mathbf{L}$ & $\mathbf{L}$ \\
\hline $\mathrm{Ma}^{29}$ & 2019 & $\mathbf{L}$ & $\mathbf{L}$ & $\mathrm{H}$ & $\mathbf{L}$ & $\mathbf{L}$ & $\mathbf{L}$ & $\mathbf{L}$ \\
\hline $\operatorname{Lier}^{30}$ & 2020 & $\mathbf{L}$ & $L$ & $\mathbf{L}$ & $U$ & $\mathbf{L}$ & $L$ & $L$ \\
\hline Wang $^{37}$ & 2020 & L & $\mathbf{L}$ & $\mathbf{L}$ & $\mathbf{L}$ & $\mathbf{L}$ & L & L \\
\hline
\end{tabular}

Figure 4. Quality Assessment of Diagnostic Accuracy Studies 2 assessment of studies. Risk of bias is designated as L, H or U. L, low; H, high; U, unclear.

outcomes inevitably reduced the quality of the present review. Furthermore, risk of bias in the flow and timing domains was frequently present. Therefore, it was not possible to perform a pooled data-analysis or meta-analysis. While case reports were included, the majority of these studies were well-designed and mostly provided their evidence clearly and accurately, according to the quality assessment using the CARE (Case report) guidelines.

\section{Discussion}

The NBI system for image-enhanced endoscopy was first conceived and developed in May 1999 and the product was launched by Olympus Corporation in May 2006 (21).

The major advantages of the NBI system are the enhancement of endoscopic visualization of superficial neoplastic lesions and their microvascular architecture. Conventional endoscopic diagnosis using WL, by contrast, is based on subtle morphological changes, e.g., superficially elevated, flat or depressed lesions, and on minimal changes in color such as reddish discoloration (19).

The present systematic review demonstrated that the application of NBI in gynecological endoscopy has the potential to improve the diagnosis of endometriosis. It may also have the potential to enhance the diagnosis of premalignant and malignant lesions in the fields of hysteroscopy, laparoscopy and colposcopy.

While Kisu et al (44) performed a review in 2012, the present systematic review is substantially updated and includes quality assessment of the studies according to the QUADAS-2 and the CARE guidelines $(6,7)$.

NBI has proven to be an efficacious approach for the diagnosis of endometrial cancer and hyperplasia, while thorough training improves the trainee's diagnostic skills to an extent depending on their previous hysteroscopic experience (37). However, it must be stressed that NBI laparoscopy was not superior in the detection of peritoneal metastases in comparison with standard WL laparoscopy alone, which was reported by Aloisi et al (39) and Schnelldorfer et al (45).

A significantly greater number of peritoneal abnormalities were identified with NBI than with standard WL. However, no statistically significant differences were observed in the identification of histologically confirmed metastatic disease. In fact, of the eight additional suspicious-appearing nodules visualized with NBI, only three were confirmed as malignant on final pathology, and none of the patients had surface 
malignancies identified with NBI that were not also seen with WL, even if in a different area (39). Aloisi et al (39) pointed out that further exploration of the use of NBI/3D WL imaging is required; this will now be further evaluated in a large randomized clinical trial with clinically relevant endpoints, with adequate power, quality control and measures (44). This will be according to the Idea, Development, Exploration, Assessment and Long-term Study (IDEAL) framework, describing 5 stages of evolution for new surgical therapeutic interventions. IDEAL is an important driver for future incremental and evidence-based modifications (46).

Furthermore, another limitation is the extension of the surgical time, particularly in laparoscopic procedures with the use of NBI. Lier et al (30) reported a median extension of surgical time of $30 \mathrm{~min}$ with NBI due to thorough inspection of the peritoneum and histological sampling. The clinical question is whether an improved detection of endometriosis with NBI/3D imaging also affects the long-term clinical outcomes after surgery, such as reintervention rates, pain-free interval and quality of life (30).

A shortcoming, particularly with the methodology reported by Barrueto et al (34), is the low specificity. This may result in unnecessary resection of healthy tissue, producing postoperative neuropathic pain and adhesion formation.

In the study by Surico et al (20), only a small number of patients were recruited, while the study was performed at a single academic institution. Furthermore, the accuracy of NBI hysteroscopy in the prediction of histological findings via analysis of interobserver variability was not assessed (20).

Wang et al (37) reported that the physician who performs hysteroscopy must be familiar with endometrial lesions, which are influenced by estrogen and progesterone secretion. Endometrial necrosis may not only be observed in malignant lesions, but also frequently appears in benign hyperplastic lesions associated with abnormal uterine bleeding. These factors increase the difficulty of hysteroscopic diagnosis; therefore, the learning curve for the diagnosis of endometrial neoplasms is relatively long. The authors report that $>200$ hysteroscopic cases are required to be performed by physicians until proficiency is reached (37).

The drawback of NBI colposcopy is that the system is expensive; thus, widespread use of it is limited and is particularly unsuitable for application in developing countries. It may be appropriate to use for educational purposes in cancer center hospitals or university hospitals (31).

In conclusion, conventional WL imaging in gynecological endoscopy is now well-established as a highly sensitive and specific technique for the diagnosis of intrauterine diseases and the present study clearly indicated that the NBI system, when applied by an expert and experienced surgeon, is capable of enhancing diagnostic accuracy. Furthermore, NBI may increase the diagnostic skills of trainees. Future directions of research should take into consideration the reoperation rates, recurrence and overall cost. Evidence-based frameworks such as IDEAL should be implemented in order to improve clinical practice. Certainly, there is a requirement for large-scale, multicenter, randomized trials to substantiate the present results as to the potential for use of NBI in gynecology, the application of which may improve patients' oncological outcomes and thus their quality of life.

\section{Acknowledgements}

The authors thank the Emeritus Professor George Iatrakis of University of West Attica (Athens, Greece) for assisting us in obtaining the full-text of articles.

\section{Funding}

No funding was received.

\section{Availability of data and materials}

All data generated or analyzed during this study are included in this published article.

\section{Authors' contributions}

All authors contributed equally to the writing and production of this manuscript. PP and KK extracted information, SS, CK, EA, PT and NA interpreted the extracted information and tabulated the data. NV checked the results and approved the study. PP was the main author that formed the conception of the study. EA checked for the eligibility of the studies. All authors read and approved the final manuscript. PP and NV confirm the authenticity of all the raw data.

\section{Ethics approval and consent to participate}

Not applicable.

\section{Patient consent for publication}

Not applicable.

\section{Competing interests}

The authors declare that they have no competing interests.

\section{References}

1. Vincent BD and Fraig M: A pilot study of narrow-band imaging compared to white light bronchoscopy for evaluation of normal airways and premalignant and malignant airways disease. Chest 131: 1794-1199, 2007.

2. Sano Y and Horimatsu T: Magnifying observation of microvascular architecture of colorectal lesions using a narrow-band imaging system. Dig Endosc 18: 44-51, 2006.

3. Watanabe A and Taniguchi M: The value of narrow band imaging endoscope for early head and neck cancers. Otolaryngol Head Neck Surg 138: 446-451, 2008

4. Machida H, Sano Y, Hamamoto Y, Muto M, Kozu T, Tajiri H and Yoshida S: Narrow-band imaging in the diagnosis of colorectal mucosal lesions: A pilot study. Endoscopy 36: 1094-1098, 2004.

5. Liberati A, Altman DG, Tetzlaff J, Mulrow C, Gøtzsche PC, Ioannidis JP, Clarke M, Devereaux PJ, Kleijnen J and Moher D: The PRISMA statement for reporting systematic reviews and meta-analyses of studies that evaluate healthcare interventions: Explanation and elaboration. BMJ 339: b2700, 2009.

6. Whiting PF, Rutjes AW, Westwood ME, Mallett S, Deeks JJ, Reitsma JB, Leeflang MM, Sterne JA and Bossuyt PM; QUADAS-2 Group: QUADAS-2: A revised tool for the quality assessment of diagnostic accuracy studies. Ann Intern Med 155: 529-536, 2011.

7. Gagnier JJ, Kienle G, Altman DG, Moher D, Sox H and Riley D; CARE Group: The CARE guidelines: Consensus-based clinical case report guideline development. J Clin Epidemiol 67: 46-51, 2014. 
8. Tulandi T and Balayla J: Study designs and the use of the canadian task force classification. J Obstet Gynaecol Can 40: 1383-1384, 2018

9. Surico D, Vigone A and Leo L: Narrow band imaging in endometrial lesions. J Minim Invasive Gynecol 16: 9-10, 2009.

10. Raimondo I, Scarciglia ML, Amadio G, Monterisi AF, Scambia G and Masciullo V: Narrow band imaging: A new diagnostic tool in adenomyosis? Int J Gynaecol Obstet 119: S531-S867, 2012.

11. Farrugia M, Nair MS and Kotronis KV: Narrow band imaging in endometriosis. J Minim Invasive Gynecol 14: 393-394, 2007.

12. Murnaghan O, Rajakumar C, Bougie O and Singh SS: Use of narrowband imaging for the surgical management of endometriosis. J Obstet Gynaecol Can 39: 711, 2017.

13. Kazama S, Hiramatsu T, Kuroda K, Hongo K, Watanabe Y Tanaka $\mathrm{T}$ and Kuriki K: A case of unique endoscopic findings of intestinal endometriosis exposed to the mucosa: Aggregation of papillary protruded bulges from the submucosal elevation of the rectum. Clin J Gastroenterol 12: 166-170, 2019.

14. Anko M, Nakamura M, Kobayashi Y, Tsuji K, Nakada S, Nakamura Y,Funakoshi T, Banno K and Aoki D: Primary malignant melanoma of the uterine cervix or vagina which were successfully treated with nivolumab. J Obstet Gynaecol Res 46: 190-195, 2020.

15. Fanfani F, Gallotta V, Rossitto C, Fagotti A and Scambia G: Narrow band imaging in borderline ovarian tumor. J Minim Invasive Gynecol 17: 146-147, 2010.

16. Fanfani F, Rossito C, Faggotti A, Gallotta V, Gagliardi ML and Scambia G: Narrow-band imaging in laparoscopic management of cervical carcinoma. J Minim Invasive Gynecol 18: 146-147, 2011.

17. Gagliardi ML, Polito S, Fagotti A, Fanfani F and Scambia G: Narrow-band imaging in laparoscopic management of recurrent platinum sensitive ovarian cancer. J Minim Invasive Gynecol 20: $10-12,2013$

18. Kobara H, Uchita K, Uedo N, Matsuura N, Nishiyama N, Kanenishi $\mathrm{K}$ and Masaki T: Uterine cervical neoplasm diagnosed by flexible magnifying endoscopy with narrow band imaging. Diagnostics (Basel) 10: 903, 2020.

19. Cicinelli E, Tinelli R, Colafiglio G, Pastore A, Mastrolia S, Lepera A and Clevin L: Reliability of narrow-band imaging (NBI) hysteroscopy: A comparative study. Fertil Steril 94: 2303-2307, 2010.

20. Surico D, Vigone A, Bonvini D, Tinelli R, Leo L and Surico N: Narrow-band imaging in diagnosis of endometrial cancer and hyperplasia: A new option? J Minim Invasive Gynecol 17: 620-625, 2010.

21. Kisu I, Banno K, Kobayashi Y, Ono A, Masuda K, Ueki A, Nomura H, Hirasawa A, Abe T, Kouyama K, et al: Flexible hysteroscopy with narrow band imaging (NBI) for endoscopic diagnosis of malignant endometrial lesions. Int J Oncol 38: 613-618, 2011

22. Kuroda K, Kitade M, Kikuchi I, Kumakiri J, Matsuoka S, Tokita S, Kuroda M and Takeda S: A new instrument: A flexible hysteroscope with narrow band imaging system: Optical quality comparison between a flexible and a rigid hysteroscope. Minim Invasive Ther Allied Technol 20: 263-266, 2011

23. Kisu I, Banno K, Susumu N, Aoki D. Magnifying hysteroscopy with narrow-band imaging for visualization of endometrial lesions. Int J Gynaecol Obstet. 115 (1): 74-5, 2011.

24. Kong L, Duan H, Zhang Y, Wang Y and Guo Y: Application of narrow-band imaging in the diagnosis of endometrial lesions. J Minim Invasive Gynecol 22: S45, 2015

25. Ozturk M, Ulubay M, Alanbay I, Keskin U, Karasahin E and Yenen MC: Using narrow-band imaging with conventional hysteroscopy increases the detection of chronic endometritis in abnormal uterine bleeding and postmenopausal bleeding. J Obstet Gynaecol Res 42: 67-71, 2016

26. Barrueto FF and Audlin KM: The use of narrowband imaging for identification of endometriosis. J Minim Invasive Gynecol 15: 636-639, 2008

27. Chandakas S, Salamalekis E and Erian J: New narrow band imaging endoscopic system for the detectionof surface pathology including endometriosis: A series of 95 patients. J Minim Invasive Gynecol 15: S1-S159, 2008.

28. Kuroda K, Kitade M, Kikuchi I, Kumakiri J, Matsuoka S, Jinushi M, Shirai Y, Kuroda M and Takeda S: Vascular density of peritoneal endometriosis using narrow-band imaging system and vascular analysis software. J Minim Invasive Gynecol 16: 618-621, 2009.

29. Ma T, Chowdary P,Eskander A, Ellett L, McIlwaine K, Manwaring J, Readman E and Maher P: Can narrowband imaging improve the laparoscopic identification of superficial endometriosis? A prospective cohort trial. J Minim Invasive Gynecol 26: 427-433, 2019.

30. Lier MC, Vlek SL, Ankersmit M, van de Ven PM, Dekker JJML, Bleeker MCG, Mijatovic V and Tuynman JB: Comparison of enhanced laparoscopic imaging techniques in endometriosis surgery: A diagnostic accuracy study. Surg Endosc 34: 96-104, 2020.
31. Fujii T, Nakamura M, Kameyama K, Saito M, Nishio H, Ohno A Hirao N, Iwata T, Tsukazaki K and Aoki D: Digital colposcopy for the diagnosis of cervical adenocarcinoma using a narrow band imaging system. Int J Gynecol Cancer 20: 605-610, 2010.

32. Nishiyama N, Kanenishi K, Mori H, Kobara H, Fujihara S, Chiyo T, Kobayashi N, Matsunaga T, Ayaki M, Yachida T, et al: Flexible magnifying endoscopy with narrow band imaging for the diagnosis of uterine cervical tumors: A cooperative study among gastrointestinal endoscopists and gynecologists to explore a novel microvascular classification system. Oncol Lett 14: 355-362, 2017.

33. Kuroda K, Kitade M, Kikuchi I, Kumakiri J, Matsuoka S, Kuroda M and Takeda S: Peritoneal vascular density assessment using narrow-band imaging and vascular analysis software, and cytokine analysis in women with and without endometriosis. J Minim Invasive Gynecol 17: 21-25, 2010.

34. Barrueto FF, Audlin KM, Gallicchio L, Miller C, MacDonald R, Alonsozana E, Johnston M and Helzlsouer KJ: Sensitivity of narrow band imaging compared with white light imaging for the detection of endometriosis. J Minim Invasive Gynecol 22: 846-852, 2015.

35. Uchita K, Kanenishi K, Hirano K, Kobara H, Nishiyama N, Kawada A, Fujihara S, Ibuki E, Haba R, Takahashi Y, et al: Characteristic findings of high-grade cervical intraepithelial neoplasia or more on magnifying endoscopy with narrow band imaging. Int J Clin Oncol 23: 707-714, 2018.

36. Tinelli R, Surico D, Leo L,Pinto V, Surico N, Fusco A, Cicinelli MV, Meir YJ and Cicinelli E: Accuracy and efficacy of narrow-band imaging versus white light hysteroscopy for the diagnosis of endometrial cancer and hyperplasia: a multicenter controlled study. Menopause 18: 1026-1029, 2011.

37. Wang W, Chen F, Kong L, Guo Y, Cheng J and Zhang Y: Prospective evaluation of the accuracy of a training program in image recognition by narrow-band imaging guided hysteroscopy of endometrial neoplasms. Gynecol Obstet Invest 85: 284-289, 2020.

38. Gallicchio L, Helzlsouer KJ, Audlin KM, Miller C, MacDonald R, Johnston $\mathrm{M}$ and Barrueto FF: Change in pain and quality of life among women enrolled in a trial examining the use of narrow band imaging during laparoscopic surgery for suspected endometriosis. J Minim Invasive Gynecol 22: 1208-1214, 2015.

39. Aloisi A, Sonoda Y, Gardner GJ, Park KJ, Elliott SL, Zhou QC, Iasonos A and Abu-Rustum NR: Prospective comparative study of laparoscopic narrow band imaging (NBI) versus standard imaging in gynecologic oncology. Ann Surg Oncol 25: 984-990, 2018.

40. Wright VC: Cervical glandular disease: Adenocarcinoma in situ and adenocarcinoma. In: Colposcopy Principles and Practice. Barbara S, Apgar GL and Spitzer M (eds). 2nd edition. Saunders Elsevier, Philadelphia, PA, 283Y310, 2008.

41. Carugno J, Di Spiezio Sardo A, Alonso L, Haimovich S, Campo R, De Angelis C, Bradley L, Bettocchi S, Arias A, Isaacson K, et al: COVID-19 pandemic. Impact on hysteroscopic procedures: A consensus statement from the global congress of hysteroscopy scientific committee. J Minim Invasive Gynecol 27: 988-992, 2020.

42. Thomas V, Maillard C, Barnard A, Snyman L, Chrysostomou A, Shimange-Matsose L and Van Herendael B: International Society for Gynecologic Endoscopy (ISGE) guidelines and recommendations on gynecological endoscopy during the evolutionary phases of the SARS-CoV-2 pandemic. Eur J Obstet Gynecol Reprod Biol 253: $133-140,2020$.

43. American Association of Gynecologic Laparoscopists. COVID-19: Joint Society Statement on Elective Surgery. Available from: http://www. aagl.org/news/covid-19-joint-statement-onelective-surgeries/.

44. Kisu I, Banno K, Tsuji K, Masuda K, Ueki A, Kobayashi Y, Yamagami W, Susumu N and Aoki D: Narrow band imaging in gynecology: A new diagnostic approach with improved visual identification (Review). Int J Oncol 40: 350-356, 2012.

45. Schnelldorfer T, Jenkins RL, Birkett DH, Wright VJ, Price LL and Georgakoudi I: Laparoscopic narrow band imaging for detection of occult cancer metastases: A randomized feasibility trial. Surg Endosc 30: 1656-1661, 2016.

46. Hirst A, Philippou Y, Blazeby J, Campbell B, Campbell M, Feinberg J, Rovers M, Blencowe N, Pennell C, Quinn T, et al: No Surgical innovation without evaluation: Evolution and further development of the IDEAL framework and recommendations. Ann Surg 269: 211-220, 2019.

This work is licensed under a Creative Commons Attribution-NonCommercial-NoDerivatives 4.0 International (CC BY-NC-ND 4.0) License. 